\title{
Pacific
}

Journal of

Mathematics

\section{FINITE GROUPS WITH A SPECIAL 2-GENERATOR} PROPERTY

Tuval S. FogueL 


\title{
FINITE GROUPS WITH A SPECIAL 2-GENERATOR PROPERTY
}

\author{
TuVal Foguel
}

This paper deals with finite groups. J. L. Brenner and James Wiegold defined a finite group $G$ as lying in $\Gamma_{1}^{(2)}$ if $G$ is nonabelian and for every $1 \neq x \in G$, either $x$ is an involution and $G=\langle x, y\rangle$ for some $y \in G$ or $x$ is not an involution and there is an involution $z \in G$ with $G=\langle x, z\rangle$. In this paper we expand the work of J. L. Brenner and James Wiegold, and that of Martin J. Evans in the investigation of which finite groups lie in $\Gamma_{1}^{(2)}$.

\section{Introduction.}

Definition 1.1. An element $x$ in a group is called a mate of an element $y \in G$ if $G=\langle x, y\rangle$.

Definition 1.2. We say that a finite group $G$ lies in $\Gamma_{1}^{(2)}$ if $G$ is nonabelian and for every $1 \neq x \in G$, either $x$ is an involution and $x$ has a mate $y \in G$ or $x$ is not an involution and $x$ has an involution $z \in G$ as a mate (see [3]).

Brenner and Wiegold [3] proved that $P S L(2, q)$ lies in $\Gamma_{1}^{(2)}$ except when $q=9$, and that $\operatorname{PSL}(n, q)$ does not lie in $\Gamma_{1}^{(2)}$ for $n \geq 3$ unless $n=3$ and $q=2$, or 4 .

Evans [6] proved that if $G=\mathrm{Sz}\left(2^{2 n+1}\right)$ is a Suzuki group, then $G$ lies in $\Gamma_{1}^{(2)}$; moreover if $G$ is a simple Chevalley group over a finite field $K$ of odd characteristic and $G$ lies in $\Gamma_{1}^{(2)}$, then $G \cong P S L(2, K)$.

Using the fact that each of the groups $S_{p}(2 n, K), P \Omega_{2 n}^{+}(K)$, and $P \Omega_{2 n}^{-}(K)$ act irreducibly on $V=K^{2 n}$, and $P S U_{m}(K)$ acts irreducibly on $V=K^{m}$, we find an element $x$ of order greater than 2 that acts trivially on a "large enough" subspace, so that two conjugates of $x$ can not act irreducibly on $V$, if dimension of $V$ is large. We get in [7] that over a field $K$ of characteristic $2, P \Omega_{2 n}^{-}(K)$, for $n \geq 4$ or $|K|>2$, and $P S U_{m}(K)$ for $n>3, S_{p}(2 n, K)$ for $n \geq 3$, and $P \Omega_{2 n}^{+}(K)$ for $n \geq 5$, or $|K|>2$, do not lie in $\Gamma_{1}^{(2)}$. Similarly we show in [7] that $\Sigma_{n}$ for $n \geq 5$ and $A_{n}$ for $n>5$ do not lie in $\Gamma_{1}^{(2)}$. 
In this paper we classify all those solvable groups that lie in $\Gamma_{1}^{(2)}$, and we show that a finite non-simple non-solvable group lies in $\Gamma_{1}^{(2)}$ if it is isomorphic to the semi-direct product of $N$ and $\langle x\rangle$ where $x$ is an involution and $N$ is a simple nonabelian group. Many simple groups are excluded from being candidates for the $N$ above. We also continue in the investigation of which simple groups lie in $\Gamma_{1}^{(2)}$.

\section{A Preliminary look at $\Gamma_{1}^{(2)}$.}

This section deals with general facts about groups which lie in $\Gamma_{1}^{(2)}$. We state Lemmas 2.1-2.6 but since they are obvious we omit the proofs.

Lemma 2.1. If $G$ is a group and $G=\langle x, y\rangle$ where $y$ is an involution and $x \neq 1$, then $H=\left\langle x, x^{y}\right\rangle$ is a normal subgroup of $G$.

Lemma 2.2. If $G$ is a nonabelian simple group and $G \leq M \leq \operatorname{Aut}(G)$, and $M=\langle x, y\rangle$ where $1 \neq x \in G, y \in M$ and is an involution, then $G=\left\langle x, x^{y}\right\rangle$.

Corollary 2.3. If $G$ is simple and $G$ lies in $\Gamma_{1}^{(2)}$, then every conjugacy class of $G$ other than the classes of elements of order 1 or 2 contains a pair of conjugate elements which generate $G$.

Lemma 2.4. If $x$ and $y$ are conjugate in $G$ and $y$ has a mate, then $x$ has a mate.

Lemma 2.5. If $G$ lies in $\Gamma_{1}^{(2)}$, then $Z(G)=1$.

Lemma 2.6. If $G$ lies in $\Gamma_{1}^{(2)}$ and $N$ is a nontrivial normal sungroup of $G$, then $G^{*}=G / N$ is cyclic.

Definition 2.7. A finite nonabelian group is called a $\Gamma$-group if $G=N P$, where $N$ is an elementary abelian normal 2-subgroup, and $P$ is a cyclic group of prime order acting irreducibly on $N$.

The last condition says that no proper subgroup of $N$ is $P$-invariant. Since $C_{N}(P)$ is $P$-invariant, $C_{N}(P)=1$ or $C_{N}(P)=N$. Since $G$ is nonabelian $C_{N}(P)=1$.

Remark 2.8. If $G$ is a $\Gamma$-group, then $p=|P|$ is an odd prime.

Remark 2.9. Given an odd prime $p$, there is a $\Gamma$-group $G$ of order divisible by $p$. It is the subgroup of $\operatorname{Aff}\left(1,2^{n}\right)$, where $n$ is the unique positive integer such that $n \mid(p-1)$, and $p \mid\left(2^{n}-1\right)$ but $p$ does not divide $2^{m}-1$ for $0<m<n$.

Lemma 2.10. $A$ Г-group $G=N P(N$ and $P$ as in Definition 2.7) has the following properties: 
1) $G$ lies in $\Gamma_{1}^{(2)}$.

2) $N$ is the commutator subgroup of $G$.

Proof. Let $y \in G-\{1\}$ and $P=\langle x\rangle$.

If $y \in N-\{1\}$, then $\langle y\rangle^{\langle x\rangle}$ is a non-trivial $P$-invariant subgroup of $N$ and $\langle y\rangle^{\langle x\rangle}=N$. Therefore, $G=\langle x, y\rangle$. If $y \in G-N$, then $y$ is $N$-conjugate to a power of $x$, so that any $\langle y\rangle$-invariant subgroup of $N$ is also $P$-invariant. Thus, $G=\langle y, n\rangle$ for any $n \in N-\{1\}$, and $G$ lies in $\Gamma_{1}^{(2)}$. Since $P$ is an abelian group, $G^{\prime} \leq N$ and $G^{\prime}=N$ because $G^{\prime}$ is $P$-invariant and nontrivial.

Notation. If $y \in G$, then $O(y)$ is the order of $y$.

Theorem 2.11. If $G$ lies in $\Gamma_{1}^{(2)}$, then $G$ has a proper normal subgroup of odd index if and only if it is a $\Gamma$-group.

Proof. By Lemma 2.10 any $\Gamma$-group lies in $\Gamma_{1}^{(2)}$, and has a proper normal subgroup of odd index.

Assume that $G$ has a proper normal subgroup $K$ of odd index. Since $|G / K|$ is odd and $|G|$ is even, $K$ is nontrivial; therefore, Lemma 2.6 gives that $G / K$ is cyclic. Let $M^{*}$ be a subgroup of $G / K$ of odd prime index $p$. If $M \leq G$ such that $M / K=M^{*}$, then $M$ is normal in $G$ and $|G / M|=p$ is an odd prime.

If $x$ is an involution in $G$, then $x$ is in $M$ because $O(x M)=1$. If $y$ is an element of $M-\{1\}$ with $O(y) \neq 2$, then $y$ has a mate $t$ of order 2. Thus, $t$ is in $M$. But $G=\langle y, t\rangle$ gives that $G=M$, a contradiction. Therefore, $|M|=2^{n}$ and $M$ is an elementary abelian 2-group. Since, $|G / K|$ is odd and $K \leq M, M=K$.

Let $x$ be an element of order $p$ in $G$, and set $P=\langle x\rangle$. Then $G$ is the semidirect product of $M$ by $P$, because $M \cap P=\{1\}$ and $G=M P$. If $z \in G-M$, then $O(z M) \neq 1$ and so $O(z M)=p$. Thus, $p \mid O(z)$ so we see that $O(z)=2^{k} p$ for some $k$. If $t=z^{p}$, then $t \in M \cap C_{G}(z)$; therefore $t$ is in $Z(G)$ and by Lemma $2.5, t=1$. Therefore, $O(z)=p$.

Let $N$ be a minimal $P$-invariant subgroup of $M$, and $d$ an element of $N-\{1\}$. Now, $d$ has a mate $y$. Since $G=\langle d, y\rangle, y$ is not an element of $M$. By the preceding paragraph $O(y)=p$. Hence, $\langle y\rangle$ is $M$-conjugate to $P$, by Sylow's Theorem. Thus, $\langle y\rangle^{m}=P$ for some $m \in M$, and because $M$ is abelian $d^{m}=d$. It follows that

$$
\left.G=\langle d, y\rangle=\left\langle d^{m}, y^{m}\right\rangle=\langle d, P\rangle=\langle N, P\rangle \text { (because } d \in N\right) .
$$

Hence, $G=N P$ and $|G|=|N| \cdot|P|$. This implies that $N=M$. Therefore $P$ acts irreducible on $M$ and $G$ is a $\Gamma$-group. 
Theorem 2.12. If $G$ lies in $\Gamma_{1}^{(2)}$ and $G \neq G^{\prime}$, then either

1) $G$ is a $\Gamma$-group,

or

2) $G$ is isomorphic to a semi-direct product of $G^{\prime}$ by $\langle y\rangle$, where $y$ is an involution. If, in this case, $G^{\prime}$ is abelian, then $G$ is isomorphic to $D_{2 p}$, where $p$ is an odd prime.

Proof. If $G^{*}=G / G^{\prime}$, then $G^{*}$ is cyclic by Lemma 2.6.

If $G^{*}$ is not a 2-group, then there exists a normal subgroup $N$ of $G$ with $G^{\prime} \leq N<G$ and $|G / N|$ is odd. Since $|G / N|>1$ is odd, $G$ is a $\Gamma$-group and $G^{\prime}=N$, by Lemma 2.10 .

If $G^{*}$ is a 2-group, note that $G$ is not a 2-group, for $Z(G)=1$. Therefore there exists an $x \in G^{\prime}$ of odd prime order $p$. The element $x$ has a mate $y$ of order 2. Since $G=\langle x, y\rangle, G^{*}=\left\langle x G^{\prime}, y G^{\prime}\right\rangle=\left\langle y G^{\prime}\right\rangle$; therefore $\left|G^{*}\right|=2$, $G^{\prime} \cap\langle y\rangle=1$, and $G$ is isomorphic to a semi-direct product of $G^{\prime}$ and $\langle y\rangle$.

If $G^{\prime}$ is abelian, then $z^{y}=z$ for any $z \in G^{\prime}$ if and only if $z=1$, because $Z(G)=1$. Hence, $y$ acts fixed point free on $G^{\prime}$ and, since $y$ is an involution, $G^{\prime}$ is of odd order and for all $z \in G^{\prime}-\{1\} z^{y}=z^{-1}$. Since $G=\langle x, y\rangle$, $G^{\prime}=\langle x\rangle$ and $\left|G^{\prime}\right|=p$. Therefore $G \cong D_{2 p}$, for $|G|=2 p$ and $G=\langle x, y\rangle$, where $x^{p}=y^{2}=1$, and $x^{y}=x^{-1}$.

Lemma 2.13. Let $G$ lie in $\Gamma_{1}^{(2)}$. If $G$ is isomorphic to a semi-direct product of $N$ by $\langle x\rangle$, where $x$ is an involution, then $x$ acts on $N$ as an outer automorphism of $N$.

Proof. Assume $x$ acts on $N$ as conjugation by an element $y \in N$, then $x y^{-1}$ acts trivially on $N$. Therefore, $1 \neq x y^{-1}$ is in the center of $G$, contradicting Lemma 2.5.

Definition 2.14. A group is a proper semi-direct product of $N$ by $P$ if $G$ is a semi-direct product of $N$ by $P$ and $N C_{G}(N) \neq G$.

Note. The situation in Lemma 2.13 gives us an example of a proper semidirect product, since $N C_{G}(N)=N \neq G$.

In [6] Evans shows that if a simple Chevalley group over a field of odd characteristic lies in $\Gamma_{1}^{(2)}$, then it is isomorphic to $\operatorname{PSL}(2, K)$. Below we will present a generalization of this result.

Definition 2.15. 1) $\Delta$ denotes a root system of a Lie algebra.

2) $\Delta^{+}$denotes the set of positive roots in $\Delta$.

3) $\Delta^{+}$denotes the set of negative roots in $\Delta$. 
4) II denotes the fundamental system of roots of $\Delta$.

5) $x_{r}(k)$ denotes the generator $\exp \left(f \operatorname{ad} e_{r}\right)$ of a Chevalley group.

6) $X_{s}=\left\langle x_{x}(k) \mid k \in K\right\rangle$.

7) $U=\left\langle x_{r}(k) \mid r \in \Delta^{+}, k \in K\right\rangle$.

Recall that each root $r \in \Delta$ can be written as $r=\sum k_{\alpha} \alpha(\alpha \in \Pi)$ with integral coefficients $k_{\alpha}$ all nonnegative or all nonpositive [10, 10.1].

Definition 2.16. The height of $r \in \Delta$ (relative to $\Pi$ ) is $h t(r)=\sum k_{\alpha}$.

From the Steinberg decomposition of automorphisms of a finite simple Chevalley group $M[4$, Theorem 12.5.1], we get that if $\tau \in \operatorname{Aut}(M)$, then $\tau=i d g f$, where $i$ is an inner automorphism, $d$ is a diagonal automorphism, $g$ is a graph automorphism, and $f$ a field automorphism. From the Bruhat decomposition [4, Chapter 8] we get that if $i \in \operatorname{inn}(M) \cong M$ since $M$ is a simple group, then $i=u_{1} h_{1} n u_{2} h_{2}$, where $u_{1}, u_{2} \in U, h_{1}, h_{2} \in H$ and $n_{t} \in N$. $H$ and $N$ denote the diagonal and monomial subgroups of $M$ respectively.

So by combining the above remarks we get that every $\tau \in \operatorname{Aut}(M)$ can be written as

$$
\tau=u_{1} h_{1} n u_{2} h_{2} d g f
$$

Theorem 2.17. Let $M$ be a non-nilpotent subgroup of a finite simple Chevalley group $G$ over a finite field $K$, and $M=\left\langle X_{s}, X_{s}^{y}\right\rangle$, where $y \in \operatorname{Aut}(G)$ and $s$ is a root of greatest height in $\Delta^{+}$, then there is a homomorphism from $S L(2, K)$ onto $M$.

Proof. Let $g$ be a graph automorphism, then since $g(r) \in \Delta^{+}$for all $r \in \Delta^{+}$, $\left.g\right|_{U}$ and $\left.g\right|_{Z(U)}$ are automorphisms.

Since $y \in \operatorname{Aut}(M)$, it follows from the remarks above that

$$
y=u_{1} h_{1} n_{t} u_{2} h_{2} d g f
$$

Let $s^{\prime}=g^{-1}(s)$, then by the choice of $s$ we get that $X_{s}$ and $X_{s^{\prime}}$ are central in $U$ [4, Theorem 5.3.3], and that $H$ [4, page 100], all diagonal automorphisms, and all field automorphisms normalize $X_{s}$ and $X_{s^{\prime}}$. So we get that

$$
\begin{gathered}
M=\left\langle X_{s},\left(X_{s}\right)^{u_{1} h_{1} n_{t} u_{2} h_{2} d g f}\right\rangle=\left\langle X_{s},\left(X_{s}\right)^{n_{t} u_{2} h_{2} d g f}\right\rangle \cong \\
\cong\left(\left\langle X_{s},\left(X_{s}\right)^{n_{t} u_{2} h_{2} d g f}\right\rangle\right)^{f^{-1} g^{-1} d^{-1} h_{2}^{-1} u_{2}^{-1}}= \\
=\left\langle\left(X_{s}\right)^{f^{-1} g^{-1} d^{-1} h_{2}^{-1} u_{2}^{-1}},\left(X_{s}\right)^{n_{t}}\right\rangle=\left\langle X_{s^{\prime}},\left(X_{s}\right)^{n_{t}}\right\rangle .
\end{gathered}
$$


Let $w_{t}$ denote the image of $n_{t}$ in the Weyl group under the natural homomorphism from $N$ to $N / H \cong W$. Since $X_{s}^{n_{t}}=X_{w_{t}(s)}, M \cong\left\langle X_{s^{\prime}}, X_{w_{t}(s)}\right\rangle$.

For all $r, s \in \Delta$ which are linearly independent there exists a $w \in W$ such that $w(r), w(s) \in \Delta^{+}$. Therefore $\left\langle X_{r}, X_{s}\right\rangle$ is isomorphic to a subgroup of $U$ which is nilpotent. Since $M$ is not nilpotent, we get that $w_{t}(s)=-s^{\prime}$. So we see that $M \cong\left\langle X_{s^{\prime}}, X_{-s^{\prime}}\right\rangle$ and by [4, Theorem 6.3.1] there is a homomorphism from $S L(2, K)$ onto $M$.

Theorem 2.18. Let $M$ be a finite simple Chevalley group over a finite field $K$ of odd characteristic $p$, and $M \leq G \leq \operatorname{Aut}(M)$. If $G$ lies in $\Gamma_{1}^{(2)}$, then $M \cong P S L(2, K)$.

Proof. Since $G$ lies in $\Gamma_{1}^{(2)}$ and $O\left(x_{s}(1)\right)=p \neq 2$ where $s$ is a root of greatest height in $\Delta^{+}, x_{s}(1)$ has a mate $y$ of order 2. By Lemma 2.2 $M=$ $\left\langle x_{s}(1), x_{s}(1)^{y}\right\rangle$, therefore $M=\left\langle X_{s}, X_{s}^{y}\right\rangle$. So by Lemma 2.16 we see that there is a homomorphism from $S L(2, K)$ onto $M$, and since $M$ is simple $M \cong P S L(2, K)$.

\section{Non-Simple Groups that lie in $\Gamma_{1}^{(2)}$.}

An easy consequence of Lemma 2.6 and Theorem 2.12 is:

Corollary 3.1. A solvable group $G$ lies in $\Gamma_{1}^{(2)}$ if and only if $G \cong D_{2 p}$, where $p$ is an odd prime, or $G$ is a $\Gamma$-group.

Theorem 3.2. If $G$ is a $n$, $n$-solvable group lying in $\Gamma_{1}^{(2)}$, then either $G$ is a nonabelian simple group or $G$ is isomorphic to a proper semi-direct product of $N$ by $\langle x\rangle$, where $x$ is an involution and $N$ is a simple group.

Proof. Let $M$ be a maximal normal subgroup of $G$ and let $G^{*}=G / M$.

Case 1: If $M=\{1\}$, then $G$ is simple.

Case 2: If $M \neq\{1\}$, then $G^{*}$ is cyclic, by Lemma 2.6 , and $G^{\prime} \neq G$. Since, any $\Gamma$-group is solvable, by Theorem 2.12 and Lemma $2.13, G$ is isomorphic to a proper semi-direct product of $G^{\prime}$ by $\langle x\rangle$, where $x$ is an involution.

If $\{1\} \neq N<G^{\prime}$ and $N$ is normal in $G$, then $G / N$ is cyclic, by Lemma 2.6. But $G / N$ is a nonabelian group; a contradiction. Therefore $G^{\prime}$ is a nonabelian characteristically simple group (since $G$ is not a solvable group); that is, $G^{\prime} \cong K_{1} \times \ldots \times K_{n}$ where $K_{i} \cong K_{j}$ are nonabelian simple groups. For $k \in K_{1}$ a nontrivial element of odd order, let $y$ be its mate of order 2. By Lemma $2.1, H=\left\langle k, k^{y}\right\rangle$ is a nontrivial normal subgroup of $G$, and $H \leq G^{\prime}$, thus $H=G^{\prime}$. Since, $K_{1}$ is a normal subgroup of $G^{\prime}$ and $G^{\prime} / K_{1}=\left\langle k^{y} K_{1}\right\rangle \cong$ $K_{2} \times \ldots \times K_{n}$ is abelian, we have, $n=1$, and so $G^{\prime}=K-1$ is a simple group. Thus by Lemma $2.13 G$ is isomorphic to the proper semi-direct product of $G^{\prime}$, a simple nonabelian group, by $\langle x\rangle$ where $x$ is an involution. 


\section{Non-Simple Non-Solvable Groups.}

In this section we look more at the structure of non-simple non-solvable groups which lie in $\Gamma_{1}^{(2)}$.

Theorem 4.1. If $G$ is isomorphic to the semi-direct product of $A_{n}$ and $\langle x\rangle$ where $x$ is an involution and $n>5$, then $G$ does not lie in $\Gamma_{1}^{(2)}$.

Proof. Suppose that $n \neq 6$, then by [11] Aut $\left(A_{n}\right)=\Sigma_{n}$. Assume that $G$ lies in $\Gamma_{1}^{(2)}$, then by Theorem $3.2 G \cong \Sigma_{n}$. It is easy to see that in $\Sigma_{n}$ $(n>4)$ the 3 -cycles do not have an involution as a mate, so $G$ does not lie in $\Gamma_{1}^{(2)}$.

Note. Note that there are two nonisomorphism classes of proper semi-direct products of $A_{6}$ and $Z_{2}$ : one is $\Sigma_{6}$ which does not lie in $\Gamma_{1}^{(2)}$, while the other involves the exceptional automorphism of $A_{6}$.

Theorem 4.2. If $G$ is isomorphic to the semi-direct product of $A_{6}$ and $\langle x\rangle$ where $x$ is an exceptional automorphism of $A_{6}$ of order 2 , then $G$ lies in $\Gamma_{1}^{(2)}$.

Proof. Let $\theta$ send the following transpositions in $\Sigma_{6}$ to products of three 2-cycles:

$$
\begin{aligned}
& (12) \longrightarrow(23)(15)(46) \\
& (23) \longrightarrow(12)(34)(56) \\
& (34) \longrightarrow(23)(16)(45) \\
& (45) \longrightarrow(34)(15)(26) \\
& (56) \longrightarrow(23)(14)(56) .
\end{aligned}
$$

Since the five 2-cycles above generate $\Sigma_{6}$ and $\theta$ acts on them as a homomorphism, and the five products of three 2-cycles above also generate $\Sigma_{6}, \theta$ is an automorphism of $\Sigma_{6}$. Since $\theta$ does not preserve the cycle structure $\theta$ is an exceptional automorphism of $\Sigma_{6}$ and $A_{6}$. It is easy to see that $\theta^{2}=1$.

Let $G=\left\langle A_{6}, \theta\right\rangle$ we will show that $G$ lies in $\Gamma_{1}^{(2)}$. Set $a=(123)=(23)(12)$, $b=a^{\theta}=(136)(254)$ (multiply left to right). Let $H=\langle a, b\rangle \leq\langle a, \theta\rangle=\langle b, \theta\rangle$, $a b=(15426)$, and $a b^{-1}=(1452)(36) \in H$, so $|H|$ is divisible by 9,5 , and 4 . Thus $H=A_{6}$, and $G=\langle a, \theta\rangle=\langle b, \theta\rangle$. Thus, elements of order 3 in $A_{6}$ have mates of order 2 in $G$.

One can check that $(12345) \in C_{G}(\theta)$. There are in $A_{6}$ two maximal subgroups that contain (12345), $M$ the stabilizer of 6 , and $N$ a transitive $A_{5}$. All elements of order 3 in $M$ are 3-cycles, while $N$ contains no 3-cycles. $\theta$ maps $M$ into $N$, and since, $M=\langle(12345),(12)(34)\rangle$,

$$
N=\left\langle(12345),(12)^{\theta}(34)^{\theta}\right\rangle=\langle(12345),(14)(56)\rangle \text {. }
$$


Since $(34)(56)(14)(56)=(143),(34)(56) \notin N$, and clearly $(34)(56) \notin M$. Thus we see that

$$
\langle(12345),(34)(56)\rangle=A_{6},
$$

and since $(12345) \in C_{G}(\theta), G=\left\langle(12345)^{i_{\theta}},(34)(56)\right\rangle$ for $i=1$ and 2 . Note that (12345) $\theta$ and $(12345)^{2} \theta$ are not conjugate in $G$. Thus, elements of order 2 in $A_{6}$ have mates in $G$.

Set $A=a b=(15426)$, and $B=b a=(25436)$, the $\theta$ sends $A \leftrightarrow b$. Set $H=\langle A, B\rangle \leq\langle A, \theta\rangle$, then $\left(A B^{3}\right)^{2}=[(16)(2435)]^{2}=(23)(45) \in H$. Since $(12345)^{(12536)}=(15426)=A$ and $(34)(56)^{(12536)}=(23)(45)$, thus, $\langle A, \theta\rangle \geq$ $\langle A,(23)(45)\rangle=A_{6}$. So, $\left\langle(15426)^{i}, \theta\right\rangle=G$ for $i=1$ and 2 . Therefore, elements of order 5 in $A_{6}$ have mates of order 2 in $G$.

By similar reasoning $A B^{3}=(16)(2435)$ has $\theta$ as a mate. Thus, elements of order 4 in $A_{6}$ have mates or order 2 in $G$. So all elements in $A_{6}$ have mates as required.

Since $\theta$ sends $b a^{-1} \leftrightarrow a b^{-1}, \theta$ inverts $a b^{-1}=(1452)(36)$. The element (24)(36) also inverts $(1452)(36)$. So, $\eta=(24)(36) \in C_{G}\left(a b^{-1}\right) . \quad \eta^{2}=$ $(24)(36)(12)(45)=(1254)(36)=\left(a b^{-1}\right)^{-1}$, since $\theta(24)(36) \theta=(12)(45)$. It follows that $\eta$ has order 8 and $\langle\eta, \theta\rangle \cong D_{8}$. Since $|\langle\eta, \theta\rangle|=16$, this group is a Sylow 2-subgroup of $G$, and all involutions outside $A_{6}$ are conjugate to $\theta$. Let $x \in G-A_{6}$, note that $x^{2} \in A_{6}$. If the' order of $x^{2}$ is odd, then $x$ is conjugate to $\theta$, or $(12345)^{i_{\theta}}$ for $i=1$ or 2 (since no elements of order 3 in $A_{6}$ commute with $\theta$ ). If $x^{2}$ has even order, then $x$ is contained in a Sylow 2-subgroup of $G$. Since $x \notin A_{6}, x$ is conjugate to $\eta$ or to $\eta^{3}$. We have seen that $\eta^{2}=(1254)(36)$, so $\eta^{2}(12)(34)=(25364)=(65432)^{2}$. Since $(65432)^{(16)(25)(34)}=(12345)$ and $(12)(34)^{(16)(25)(34)}=(34)(56)$, we get $\left\langle\eta^{i},(12)(34)\right\rangle=G$ for $i=1$ or 3 .

Proposition 4.3. Let $M$ be a finite simple Chevalley group over a finite field $K$ of characteristic $p$, and let $G$ be isomorphic to the semi-direct product of $M$ and $\langle x\rangle$ where $x$ is an involution. If $G$ lies in $\Gamma_{1}^{(2)}$, then $M \cong P S L(2, K)$.

Proof. This is a direct consequence of Theorem 2.18.

Note. Note that since $A_{5} \cong P S L(2,5)$, it is not true that for every $M=$ $P S L(2, K)$ where $K$ is a finite field of odd characteristic $p$, there exists a proper semi-direct product $G$ of $M$ and $\langle x\rangle$ where $x$ is an involution such that $G$ lies in $\Gamma_{1}^{(2)}$.

In [7, Chapter 8] we eliminate many more simple groups from the possibilities for $N$ in Theorem 3.2. 


\section{5. $E_{8}$ Case.}

The Lie algebra $L$ over a field $K$ has a Cartan decomposition [4, Ch.7] $L=H \oplus \sum_{r \in \Delta} L_{r}$, where $H$ is generated by $h_{r}$ for $r \in \Pi$, and $L_{r}$ is a onedimesional vector space generated by an element $e_{r}$ for $r \in \Delta$. For a simple Lie algebra $L$ over $K$, Chevalley defined a group $L(K)$ which is simple except for a few exceptional cases. $L(K)$ is uniquely determined by its action on $\left\{h_{q}, e_{r}\right\}_{r \in \Delta, q \in \Pi \text {. }}$

The elements of $\Pi$ will be denoted by $\alpha_{1}, \ldots, \alpha_{1}$. The method of this section gives us some insight into the action of $L(K)$ on $L$ and proves that $L(K)$ does not lie in $\Gamma_{1}^{(2)}$ when $\Delta=E_{8}$. In this section we are using the standard notation for roots in $E_{8}$.

Definition 5.1. Let $f$ be a function from $\Pi \cup \Pi^{-}$to the integers defined by $f\left(\alpha_{i}\right)=f\left(-\alpha_{i}\right)=i-1$.

Definition 5.2. $\Psi=\left\{\tau \in \Delta \mid\left(\tau, \alpha_{1}\right)=0\right\}$, where $($,$) is as in [10, page 39].$

Lemma 5.3. If $\Delta=E_{8}$, and $\tau \in \Psi$, then $x_{\alpha_{1}} e_{\tau}=e_{\tau}$, and $x_{-\alpha_{1}} e_{\tau}=e_{\tau}($ where $\left.x_{r}=x_{r}(1)\right)$.

Proof. Since all roots in $E_{8}$ have the same length, this implies that if $\tau \in \Psi$, $\tau \neq-\alpha_{1}$ and $\tau-\alpha_{1}$ and $\tau+\alpha_{1} \notin \Delta$. Thus by the formula on [4, page 61] we see that $x_{ \pm \alpha_{1}} e_{\tau}=e_{\tau}$.

Let $L=H \oplus \sum_{t \in \Delta} L_{t}$ be the Cartan decomposition of $L$ [4, Ch.3]. $L$ can be written as $L=\sum_{t \in \Pi} H_{t} \oplus \sum_{t \in \Delta} L_{t}$ where $H_{t}=\left\langle h_{t}\right\rangle$, and $L_{t}=\left\langle e_{t}\right\rangle$. For $\Delta=E_{8}$, let $T=H_{\alpha_{1}} \oplus H_{\alpha_{2}} \oplus H_{\alpha_{3}} \oplus \sum_{t \in \Delta-\Psi} L_{t}$ and,

$$
L^{\prime}=\sum_{t \in \Pi-\left\{\alpha_{2}, \alpha_{3}\right\}} H_{t} \oplus \sum_{t \in \Psi} L_{t} .
$$

Note. Note that $L=L^{\prime} \oplus T$, and $x_{\alpha_{1}}$ and $x_{-\alpha_{1}}$ act trivially on $L^{\prime}$.

Lemma 5.4. Given $L, L^{\prime}$ and $T$ as before, then there exists no subset $0 \neq$ $S \leq L^{\prime} \cap H$ invariant under the action of $E_{8}(K)$ where $K$ is a finite field of characteristic 2.

Proof. Assume that such an $S$ exists, then let $x \in S-\{0\}$. We can write $x$ as $x=a_{1} h_{\tau_{1}}+\ldots+a_{n} h_{\tau_{n}}$ where each $a_{i} \neq 0$, each $\tau_{i} \in \Pi$, and $f\left(\tau_{1}\right)<\ldots<$ $f\left(\tau_{n}\right)$.

Note that in all cases, $f\left(\tau_{1}\right)>2$ by the way we constructed $L^{\prime}$. Thus we can choose a root $t \in \Pi$ with $f(t)=f\left(\tau_{1}\right)-1$ such that $\left\langle t, \tau_{1}\right\rangle=$ 
$2\left(t, \tau_{1}\right) /(t, t)=1(\bmod 2)$, and $\left\langle t, \tau_{i}\right\rangle=0$ for $i=2, \ldots, n$. So we get that $x_{t} h_{\tau_{1}}=h_{\tau_{1}}+e_{\tau}$, and $x_{t} h_{\tau_{i}}=h_{\tau_{i}}$ for $i=2, \ldots, n$. Therefore $x_{t} x \notin S$, contradicting that $S$ is $L(K)$-invariant. Therefore there exists no subset $0 \neq S \leq L^{\prime} \cap H$ invariant under the action of $L(K)$.

Lemma 5.5. There is no invariant subspace of $L^{\prime}$ under the action of $E_{8}(K)$ where $K$ is a finite field of characteristic 2.

Proof. In view of Lemma 5.4 it will be sufficient to prove that any $E_{8}(K)$ invariant nonzero subspace $S$ of $L^{\prime}$ is a subspace of $H$.

Assume that $S$ is not a subspace of $H$, then we can write any element $v \in S-H$ as $v=a_{s_{1}} e_{s_{1}}+a_{s_{2}} e_{s_{2}}+\ldots+h$, where $h \in H$, and $s_{i} \in \Psi$.

Since all the roots in $E_{8}$ have the same length, then by [10, 10.4 Lemma C] all the roots are conjugate under $W$, the Weyl group of $E_{8}$. Thus there exists an element $w \in W$, with $w\left(s_{1}\right)=\alpha_{1}$. Note that since $w$ is an automorphism $w\left(s_{i}\right) \neq \alpha_{1}$ for $i>1$, and that $w=w_{r_{1}} \ldots w_{r_{t}}$ (here $w_{r_{i}}$ is the reflection in the hyperplane orthogonal to the root $r_{i}$ ). Let $N$ be the monomial subgroup of $E_{8}(K)$, and $n_{r}$ as in [4, Proposition 6.4.2]. By [4, Theorem 7.2.2] there is a homomorphism from $N$ onto $W$ under which $n_{r} \longrightarrow w_{r}$ for all $r \in \Delta$. $n_{r}$ acts invariantly on $H$, and $n_{r} e_{s}= \pm e_{w_{r}(s)}$. Since we are in characteristic 2, $n_{r} e_{s}=e_{w_{r}(s)}$. Let $n=n_{r_{1}} \ldots n_{r_{t}} \in N$, then $n \longrightarrow w$ by the homomorphism $N \longrightarrow W$. So $n e_{s_{1}}=e_{w\left(s_{1}\right)}=e_{\alpha_{1}}$, and $n h \in H$. Hence $n v$ contains a term $e_{\alpha_{1}}$, contradicting that $v \in S$. Thus $S$ is a subspace of $H$.

Theorem 5.6. If $G=E_{8}(K)$ where $K$ is a finite field of characteristic 2, then $G$ does not lie in $\Gamma_{1}^{(2)}$.

Proof. Using the description of roots in the system $E_{8}$ in terms of orthogonal vectors given in [2, page 268] we get that the number of positive roots in $\Psi$ is

$$
1+6+6+\left(\begin{array}{l}
6 \\
2
\end{array}\right)+\left(\begin{array}{l}
6 \\
2
\end{array}\right)+\left(\begin{array}{l}
6 \\
3
\end{array}\right)=63
$$

So we get that $\operatorname{cod}\left(L^{\prime}\right)=117$. Both $x_{\alpha_{1}}$ and $x_{-\alpha_{1}}$ act trivially on $L^{\prime}$; therefore $x_{\alpha_{1}} x_{-\alpha_{1}}$ also acts trivially on $L^{\prime}$. Assume that $G$ lies in $\Gamma_{1}^{(2)}$, then since $O\left(x_{\alpha_{1}} x_{-\alpha_{1}}\right) \neq 1$ or $2, x_{\alpha_{1}} x_{-\alpha_{1}}$ has a mate of order 2 . Let $S=L^{\prime} \cap\left(L^{\prime}\right)^{y}$; since $G=\left\langle x_{\alpha_{1}} x_{-\alpha_{1}}, y\right\rangle$, we see that $G$ acts invariantly on $S$. Since

$$
\operatorname{cod}(S) \leq 2 \operatorname{cod}\left(L^{\prime}\right)=234<248
$$

$S \neq 0$ contradicting Lemma 5.5 . 


\section{Twisted Groups in odd Characteristic.}

Lemma 6.1. If $q$ is odd and $G={ }^{2} A_{l}\left(q^{2}\right)$ for $l \geq 2$, or ${ }^{2} D_{l}\left(q^{2}\right)$ for $l \geq 4$, or ${ }^{2} E_{6}\left(q^{2}\right)$, then $|G|>\left|S L_{2}\left(q^{2}\right)\right|$. Also $\left|{ }^{3} D_{4}\left(q^{3}\right)\right|>\left|S L_{2}\left(q^{3}\right)\right|$ for $q$ odd.

Proof. For $q=p^{n}$ where $p$ is an odd prime we get:

1) $\left|S L_{2}\left(q^{2}\right)\right|=q^{2}\left(q^{4}-1\right)<(1 / 3) q^{3}\left(q^{2}-1\right)\left(q^{3}+1\right) \leq\left|{ }^{2} A_{l}\left(q^{2}\right)\right|, l \geq 2$.

2) $\left|S L_{2}\left(q^{2}\right)\right|=q^{2}\left(q^{4}-1\right)<(1 / 4) q^{12}\left(q^{4}-1\right)<\left|{ }^{2} D_{l}\left(q^{2}\right)\right|, \quad l \geq 4$.

3) $\left|S L_{2}\left(q^{2}\right)\right|=q^{2}\left(q^{4}-1\right)<(1 / 3) q^{36}\left(q^{5}+1\right)<\left|{ }^{2} E_{6}\left(q^{2}\right)\right|$.

4) $\left|S L_{2}\left(q^{3}\right)\right|=q^{3}\left(q^{6}-1\right)<q^{12}\left(q^{6}-1\right)<\left|{ }^{3} D_{4}\left(q^{3}\right)\right|$.

Consider a Chevalley group $G^{*}=L(K)$ and $\rho$ a non-trivial symmetry of the Dynkin diagram for $L$. Recall that if $K$ has a certain order depending on $L$, then we can choose an automorphism $\sigma$ (a product of a field automorphism and a graph automorphism determined by $\rho$ ) of $G^{*}$ such that the twisted group $G={ }^{i} L(K)$ is a subgroup of $G^{*}$ stabilized by $\sigma$, and $U^{1}$ is the subgroup of $U$ centralized by $\sigma$ (recall that $U^{1} \leq G$ ). We will call $G^{*}$ the corresponding Chevalley group of $G$. Note that $\sigma\left(x_{r}(t)\right)=x_{r^{\prime}}\left(\gamma_{r} t^{\prime}\right)$, where $\gamma_{r}= \pm 1$ and $t^{\prime}=f(t), f$ a certain field isomorphism, and $r^{\prime}$ arises from the symmetry of the Dynkin diagram $[4,12.2]$. Since all the roots in the system associated with the groups of Lemma 6.1 have same length, the above action is an isometry [4, Prop. 12.2.2], and there exists a unique root of maximal length $s[10,10.4$ Lemma $\mathrm{A}]$, and thus $s^{\prime}=s$ (note that this is not the case for $\left.{ }^{2} G_{2}\left(3^{2 m+1}\right)[4,12.4]\right)$.

Lemma 6.2. If $G$ is a simple twisted group, and $G^{*}$ is the corresponding Chevalley group of $G$, then $\operatorname{Aut}(G) \leq \operatorname{Aut}\left(G^{*}\right)$.

Proof. From [8, page 303 and 5, Table 5] Aut $(G)$ is a product of an inner, a diagonal, and a field automorphism.

Theorem 6.3. If $q=p^{n}$ where $p$ is an odd prime and $G$ is a simple group of type ${ }^{2} A_{l}\left(q^{2}\right), l \geq 2$, or ${ }^{2} D_{l}\left(q^{2}\right), l \geq 4$, or ${ }^{2} E_{6}\left(q^{2}\right)$, or ${ }^{3} D_{4}\left(q^{3}\right)$, then $G$ does not appear as a composition factor of any group in $\Gamma_{1}^{(2)}$.

Proof. Let $K=G F\left(q^{2}\right)$ if $G={ }^{2} A_{l}\left(q^{2}\right), l \geq 2$, or ${ }^{2} D_{l}\left(q^{2}\right), l \geq 4$, or ${ }^{2} E_{6}\left(q^{2}\right)$, and $K=G F\left(q^{3}\right)$ if $G={ }^{3} D_{4}\left(q^{3}\right)$. Let $s$ be the root of maximal height. If $\gamma_{s}=1$, let $x=x_{s}(1)$. If $\gamma_{s}=-1$ (this can happen in the case that 
$K=G F\left(q^{2}\right)$ ), then the automorphism $f$ associated with the group has order 2 and there exists an element $t \in K-\{0\}$ such that $t^{\prime}=-t$. So, let $x=x_{s}(t)$. In each case $\sigma(x)=x$ and $x \in Z(U) \cap U^{1}$ [4, Def. 13.4.2] so $x \in Z\left(U^{1}\right)$ and $O(x)=p$.

If $G$ appears as a composition factor of any group in $\Gamma_{1}^{(2)}$, then $x$ has a mate $y \in \operatorname{Aut}(G) \leq \operatorname{Aut}\left(G^{*}\right)$ of order 2 , and $G=\left\langle x, x^{y}\right\rangle \leq\left\langle X_{s},\left(X_{s}\right)^{y}\right\rangle=M$ (note that $M$ is a subgroup of $G^{*}$, not of $G$ ) from Lemma 2.2. Since $M$ is a nonnilpotent subgroup of $G^{*}$ by Lemma 2.17, there is a homomorphism from $S L(2, K)$ onto $M$, but by Lemma $6.1|G|>|M|$, a contradiction.

\section{Centralizers and $\Gamma_{1}^{(2)}$.}

In this section we will use the result that if two subgroups have order greater that the square root of the order of the group, then they have a nontrivial intersection [9, Sec. 2.5], to investigate whether some groups lie in $\Gamma_{1}^{(2)}$.

Lemma 7.1. If $G$ is a finite simple group with an element of order not 1 or 2 that has a centralizer of order larger $(|G|)^{1 / 2}$, then $G$ does not appear as a composition factor of any group in $\Gamma_{1}^{(2)}$.

Proof. Assume that $G$ appears as a composition factor of a group $H$ in $\Gamma_{1}^{(2)}$. Then, by previous results, $G$ is a normal subgroup of $H$ with index at most two. Let $x \in G$ such that $O(x) \neq 1$ or 2 and $\left|C_{G}(x)\right|>(|G|)^{1 / 2}$. Since $H$ lies in $\Gamma_{1}^{(2)}, x$ has a mate $y \in H$ of order 2. By Lemma $2.2 G=\left\langle x, x^{y}\right\rangle$, and since $\left|C_{G}\left(x^{y}\right)\right|=\left|C_{G}(x)\right|$ from the above we get that $Z(G) \neq 1$, contradicting the simplicity of $G$.

Theorem 7.2. The groups $J_{2}, S u z, C o_{1}, L y, F i_{22}, F i_{23}, F i_{24}^{\prime}, D_{4}(2)$, and ${ }^{2} D_{4}(2)$ do not appear as a composition factor of any group in $\Gamma_{1}^{(2)}$.

Proof. From [5] we see that each of the above groups has an element of order 3 with a centralizer of order greater than the square root of the order of the group.

\section{References}

[1] L.B. Beasly and J.L. Brenner, Two-generator groups IV, Congressus Numeratium, 53 (1986), 95-112.

[2] N. Bourbaki, Groupes et Algebres de Lie, IV, V, VI, Hermann, Paris 1968.

[3] J.L. Brenner and James Wiegold, Two-generator groups I, Michigan Math. J., 22 (1975), 53-64.

[4] Roger W. Carter. Simple groups of Lie type, Wiley-Interscience Publishers, New York, 1989. 
[5] J.H. Conway, R.T. Curtis, S.P. Norton, R.A. Parker and R.A. Wilson, Atlas of Finite Groups, Oxford University Press, Oxford, New York, Toronto, 1985.

[6] Martin J. Evans, A note on two-generator groups, Rocky Mountain Journal of Mathematics, 17 No.4 (1987), 887-889.

[7] Tuval Foguel, Finite Groups with a Special 2-generator property, and Order of Centralizers in Finite Groups, Thesis, University of Illinois at Urbana-Champaign, 1992.

[8] Daniel Gorenstein, Finite Simple Groups, An Introduction to their Classification, Plenum Press, New York and London, 1982.

[9] I.N. Herstein, Topics in Algebra, Wiley Publisher, New York, 1975.

[10] James E. Humphreys, Introduction to Lie algebras and Representation Theory, Springer Verlag, Berlin, Heidelberg, New York, 1987.

[11] Michio Suzuki, Group Theory I, Springer-Verlag, Berlin, Heidelberg, New York, 1982.

Received March 22, 1993 and revised November 30, 1993. Parts of the contents of this paper are from the author's thesis carried out in the University of Illinois at UrbanaChampaign under the supervision of Professor Michio Suzuki. The idea of looking at centralizers, used in Section 7, was suggested to me by Professor John H. Walter. I would like to thank the referee for his comments and suggestions. Research at MSRI supported in part by NSF grant \#DM 9022140.

The UNIVERSITY OF THE WeST INDIES

MONA, KINGSTON 7

JAMAICA

E-MAIL ADDRESS: foguel@uwimona.edu.jm 



\section{CONTENTS}

N. Ben Amar, Tangential deformations on the dual of nilpotent special Lie

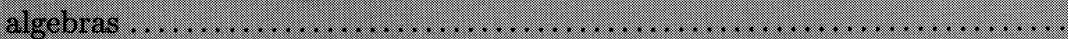

Martin Bendersky, Donald M. Davis and Mark Mahowald, $v_{1}$-periodic

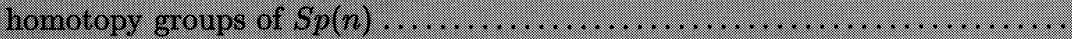

Georgia Benkart, Seok-Jin Kang, Kailash C. Misra, Indefinite Kac-

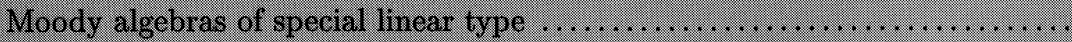

Robin Brooks and Charles Odenthal, Nielsen numbers for roots of maps

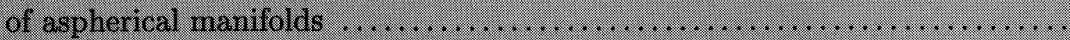

L.J. Bunce and J.D. Maitland Wright, Velocity maps in von Neumann

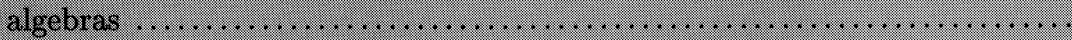

Bradley N. Currey, Smooth decomposition of finite multiplicity monomial representations for a class of completely solvable homogeneous spaces ...... R.J. Daverman and D.F. Snyder, On proper surjections with locally triv-

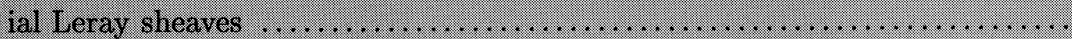

Patrick N. Dowling, Zhibao Hu and Mark A. Smith, MLUR renormings

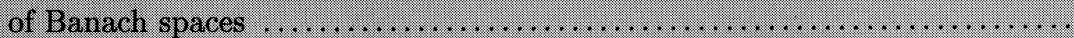
Tuval Foguel, Finite groups with a special 2-generator property ..........

Mourad E.H. Ismail and Mizan Rahman, Some basic bilateral sums and

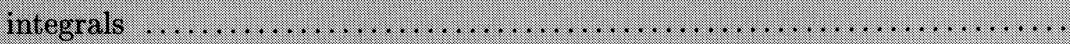

Wojciech Jaworski, Strong approximate transitivity, polynomial growth, and spread out random walks on locally compact groups $\ldots \ldots \ldots \ldots \ldots . . . . . .$.

N. Kutev and F. Tomi, Nonexistence and instability in the exterior Dirichlet problem for the minimal surface equation in the plane............

A. Nobile, Equisingularity Theory for Plane Curves With Embedded

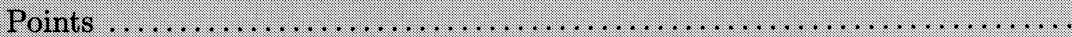

Dominikus Noll, Directional differentiability of the metric projection in Hilbert space 


\section{PACIFIC JOURNAL OF MATHEMATICS}

Volume $170 \quad$ No. $2 \quad$ October 1995

Tangential deformations on the dual of nilpotent special Lie algebras

297

NABIHA BEN AMAR

$v_{1}$-periodic homotopy groups of $S p(n)$

319

MaRTIN BENDERSKY, DONALD M. DAVIS and MARK MAHOWALD

Indefinite Kac-Moody algebras of special linear type

GeOrgia BenKart, SEOK-Jin KANG and KaILASh C. MisRa

Nielsen numbers for roots of maps of aspherical manifolds

405

ROBIN B. S. BROOKS and CHARLES ODENTHAL

Velocity maps in von Neumann algebras

L. J. BUNCE and JOHN DAVID MAITLAND WRIGHT

Smooth decomposition of finite multiplicity monomial representations for a class of 429 completely solvable homogeneous spaces

\section{BRADLEY CURREY}

On proper surjections with locally trivial Leray sheaves

ROBERT JAY DAVERMAN and DAVID FRED SNYDER

MLUR renormings of Banach spaces

PATRICK DOWLING, Zhibao Hu and MARK ANDREW SMIth

Finite groups with a special 2-generator property

TUVAL S. Foguel

Some basic bilateral sums and integrals

MOURAD ISMAIL and MIZAN RAHMAN

Strong approximate transitivity, polynomial growth, and spread out random walks on 517 locally compact groups

WOJCIECH JAWORSKI

Nonexistence and instability in the exterior Dirichlet problem for the minimal surface 535 equation in the plane

NiKolai KuteV and FriedRich TOMI

Equisingularity theory for plane curves with embedded points

Augusto Nobile

Directional differentiability of the metric projection in Hilbert space

DOMINIKUS NOLL 